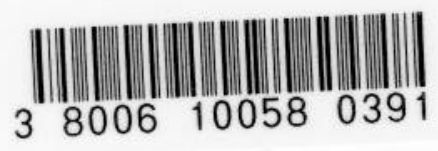

REPORT NO. 19

August, 1948

\section{THE COLLEGE OF AERONAUIICS,}

\section{CR A N F IEID}

Flutter of Systems with Many Freedoms

$$
- \text { by- }
$$

W.J. Duncan, D.Sc., F.R.S.,

Professor of Aerodynamics at the College of Aeronautics, Cranfield.

$--\infty 00--$

\section{SUMMARY}

Experience has shown that it is often necessary to retain many degrees of freedom in order to calculate critical flutter speeds reliably, but this entails much labour. Part I discusses the choice of a minimum set of freedoms and suggests that this should be based on the equation of energy and the use of the Lagrangian dynamical equation corresponding to any proposed additional freedom. The methods for conducting flutter calculations so as to minimise labour are treated in Part II. 


\section{CONTENTS}

1. Introduction

2. Some Exactly Soluble Problems of the Flutter of Elastic Wings. $\ldots$... 4

\section{Part I}

\section{Choice of the Degrees of Irreedom}

3. Types of Generalised Coordinates ... ... 5

4. The Equation of Energy $\quad \ldots \quad \ldots . \quad \ldots \quad 5$

5. General Discussion of Couplings $\ldots$... 8

6. Comparison of Inertial and Aerodynamic

Couplings. $\ldots . \quad \ldots \quad \ldots \quad \ldots \quad \ldots 11$

7. Principles Governing the Choice of

Freedoms $\quad \ldots . \quad \ldots \quad \ldots . \quad \ldots \quad \ldots 11$

8. Illustrative Examples on the Choice of

Freedoms

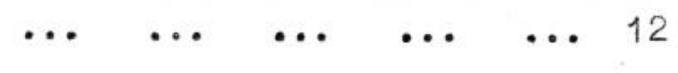

\section{Part II}

Methods for Predicting Flutter

Characteristics

9. Non-Dimensional Form of the Equations

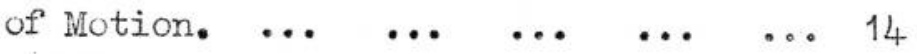

10. The Orthodox Method of Solution. ... ... 15

11. The Method of R.A. Frazer $\ldots$... $\quad \ldots \quad 16$

12. Methods Based on the Use of Matrices ... 16

13. The Method of Duncan, Collar and Lyon. ... 16

14. Another Inverse Method. $\ldots . \quad \ldots \quad \ldots 17$

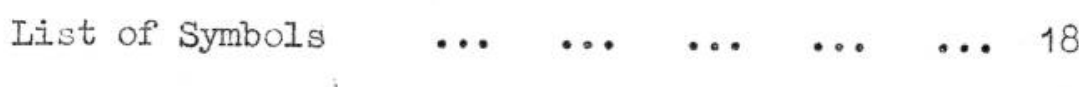

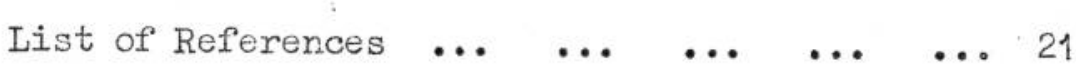




\section{Introduction}

The aim of this paper is to discuss methods for calculating the criticul flutter speeds and the nature of the motion at these speeds for systems with a large number of degrees of freedom. This problem is becoming increasingly important since it is now recognised that reliable estimates of critical speeds can, in many instances, only be made when many independent kinds of motion of the structure are admitted. However, the labour in the calculations increases exceedingly rapidly as the number of degrees of freedom is increased. Hence two principal problems arise:-

(a) The choice of a minimum set of dynamical coordinates or degrees of freedom which leads to calculated results of adequate accuracy.

(b) The choice of the method of conducting the calculations after the dynamical coordinates have been chosen. the paper.

These problems are separately considerea in Parts I and II of

It is concluded that a particular freedom $F$ must be retained when the balance of energy at a critical flutter speed is sensitive to its inclusion, unless it can be shown that the amplitude of $F$ is very small. This amplitude will be very small when one or both of the following conditions is satisfied:-

(a) The coupling terms in the Lagrangian dynamical equation corresponding to $F$ are all very sma.1.

(b) The impedance for $F$ at the critical flutter speed and for the flutter frequency is very large.

As regards the energy balance, it is shown that large skew symmetric components in the aerodynamic stiffnesses are of particular importance. Inverse methods appear to be the most advantageous for the calculation of critical flutter speeds when there are many freedoms,

There are a few known special cases where 'exact: calculations of critical flutter speeds can be made for elastic continuous systems having infinitely many degrees of freedom. Such systems throw much light on the general problem of the choice of freedoms and they are briefly considered in the following section. 


\section{Some Exactly Soluble Problems of the Flutter of Elastic Wings}

Exact solutions of two problems on the flutter of continuous elastic cartilever wings have already been published and the details are as follows:-

(a) Uniform rectangular wing supported by a pair of uniform parallel spars. The covering has no proper torsional stiffness and both the flexural and torsional stiffnesses are provided by the uniform spars. The inertial and aerodynamic coefficients are independent of spanwise position (See references 1, 3 and 4).

(b) Uniform rectangular wing as for case (a) but now the torsional stiffness is provided by the uniform covering or by a torque tube and the Plexural stiffness is assumed to be negligible $e^{\text {it }}$ (See the Appendix to reference 7).

Both wings have the following special dynamical characteristics. The modes of oscillation in vacuo or in an airstream occur in pairs which, in general, have both flexural and tcrsional components. The displacements in both flexure and torsion for the rth pair are proportional. to the displacements in the rth mode of the purely flexural oscillation of a uniform cantilever beam for case (a) and to the displacements in the rth mode of the purely torsional oscillations of a uniform cantilever for case (b). Wher the wings are exposed to an air current the two modes of the rth pair become coupled together aerodynamically, but remain uncoupled to all other modes. The flutters. which occur are therefore effectively binary and the earliest flutter occurs in the modal pair corresponding to the fundamental free mode.

These results are not, in fact, isolated and other similar cases will be discussed in a separate paper. At present we wish to emphasise the remarkable simplicity of the solutions, which were obtained in an orthodox way by solving the differential equations of the problems. An independent explanation of the results is given in $\$ 6$ by means of an' analysis of the various couplings between the modes.

\section{$/ \underline{P A R T} I \cdots \cdots$}

zx

This is of course an artificial case but is not without interest since the flexural stiffness does not greatly influence the flutter.

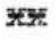

Or $(r-1) \frac{\text { th }}{}$ overtone. 


\section{PART: I}

\section{CHOICT OF THE DEGREES OF HREEDOM}

\section{Types of Generalised Coordinates}

The generalised coordinates should be chosen so as to secure some advantage of simplicity or convenience. There appear to be two particularly advantageous types:

(a) Those for which the specification of the displacements is specially simple.

Examples. The purely flexural and purely torsional coordinates of the classical theory of wing fllutter.

(b) Those which secure the greatest simplification of the dynamical equations, as by the vanishing of certain coefficients.

Examples. Normal coordinates for which the inertial and elastic coupling coefficients vanish.

In the further discussion we shall adopt normal coordinates for the main structural deformations. These correspond, strictly, to the free modes of oscillation of the structure in vacuo, but differ little from the modes of oscillation in still aix. These must be supplemented by certain general rigid body freedoms, for the resultant force and moment on the system are not, in general, zero when the oscillations occur in an airstream. Lastly, there will be one coordinate ror the angular movement of each control surface which is concerned in the flutter. The main problem is to decide which of the nomal modes and genergl sreedoms must be retained in the calculations.

For a conventional aircraft, having a foremand-aft plane of symetry, and in straight symmetric filight, the small oscillations of symmetric and antisymmetric types will be independent. For each type only the corresponding normal modes, general freedoms and control movements must be retained. For example, in symetric flutter a single symmetrically placed ruddex takes no part and the body freedoms are pitching, normal displacement and fore-and-aft displacement; the latter will usually be negligible.

In order to determine what normal coordinates are to be retained the influence of an added coordinate on the energy balance must be examined. We accordingly pass next to the equation of energy.

\section{The Equation of Energy}

For the sake of conciseness and perspicuity we shall use the matrix notation. Thus the whole set of the equations of free motion will be written

$$
\mathrm{A} \ddot{q}+\mathrm{B} \hat{q}+\mathrm{Cq}=0
$$


where A, B, C are the square matrices of the inertias, damping coefficients and stiffnesses, respectively, and $q$ is the colum matrix of the generalised coordinates. $A, B$ and $C$ are independent of the generalised coordinates and of tirne. The equation of energy. is obtained by premultiplying the last by ' $\dot{q}, \dot{i} \cdot e_{e}$, by the row matrix whose elements are the generalised velocities, and it is accordingly

$$
\dot{q}^{\prime} A \ddot{q}+\dot{q}^{\prime} B \dot{q}+\dot{q}^{\prime} C q=0 \text {. }
$$

Now $A$ is always symmetric and the kinetic energy of the system is

$$
T=\frac{1}{2} \dot{q}^{t} A \dot{q},
$$

a quadratic form in the generalised velocities. Therefore

$$
\frac{d T}{d t}=\frac{1}{2} \dot{q}^{\prime} A \ddot{q}+\frac{1}{2} \ddot{q}^{\prime} A \hat{q}
$$

since $A$ is independent of time. But

$$
\dddot{q}^{\prime} A \stackrel{q}{=}=\dot{q}^{\prime} \cdot \mathscr{q}
$$

by transposition, since $A^{\prime}=A$, and we obtain

$$
\frac{d T}{d t}=\dot{q}^{\prime} A \ddot{q} \text {. }
$$

For a system subject to aerodynamic actions neither $B$ nor $C$ is, its general, symmetric. We may always put

$$
\begin{aligned}
& B=B_{1}+B_{2} \\
& C=C_{1}+C_{2}
\end{aligned}
$$

where the suffixes 1,2 are applied to symmetric and skew-symnetric matrices respectiveiy. Then

$$
\begin{aligned}
& B_{1}=\frac{1}{2}\left(B+B^{\prime}\right) \\
& B_{2}=\frac{1}{2}\left(B-B^{\prime}\right)
\end{aligned}
$$
and there are similar expressions for $C_{1}$ and $C_{2}$. Tet the dissipation
function be

$$
=\frac{1}{2} \dot{q}^{\prime} B \dot{q}=\frac{1}{2} \dot{q}^{\prime} B_{1} \dot{q}
$$

since

$$
x^{t} Y X=0
$$
when $Y$ is skew-symuetric and $x$ is arbitrary. Also let the "potential"
be

$$
V^{\prime}=\frac{1}{2} q^{2} C_{1} q
$$

so that

$$
\frac{d V}{d t}=\dot{q}^{\prime} C_{1} q
$$

The

w An accent appended to the symbol for a matrix indicates that its transposed is to be taken. 
The equation of energy (2) can now be written

$$
\frac{d}{d t}(\gamma+y)+2 \bar{r}+\dot{q}^{\prime} C_{2} q=0 .
$$

The final term in the last equation is of great importance and must be discussed in detail.

The equation of energy is valid for any free motion of the system, but particularly illuminating deductions can be drawn wher the motion consists of a single constituent, corresponding either to a single real root or to a conjugate complex pair of roots of the determinantal equation.

Case (2) Roal exponential motion (Divergence or subsidence).

Here we have

$$
q=q_{0} e^{\lambda t}
$$

with $\lambda$ real and $q_{0}$ a colurm of real constants. Then

$$
\dot{q}=\lambda q
$$

and $q^{\prime} C_{2} q=\lambda q^{\prime} C_{2} q=0$

by equation (8). Therefore the equation of energy (11) becomes

$$
\frac{d}{d t}(T+V)+2 t=0
$$

If $F$ is negative, obviously the motion can grow. But suppose now that is positive during the motion ${ }^{2}$. Then we get

$$
\frac{d}{d t}(T+V)<0
$$

at all instants. So long as $\mathcal{V}_{\text {is }}$ essentially positive this implies decay of the motion. However, is not wholly of elastic origin and at higher values of the relative wind speed it may cease to be essentially positive. Then may increase, i.e., the motion may grow, through $\frac{\mathrm{d} \mathbf{V}}{\mathrm{dt}}$ being

F and " $\mathrm{F}$ are zero and the equation of energy becomes

$$
W=\text { constant }
$$

but the displacements need not be zero as they can be supposed to grow exceedingly slowly to finite values. This equation can be made the basis for calculating the critical divergence speeds, but we shall not pursue this question here.

Case (b)

35 When the values of the aerodynamic damping coefficients for divergent motion as given by two-dimensional vortex sheet theory are used, the condition that the dissipation function shall be essentially positive is not satisfied for any value of the non-dimensional divergence parameter $\lambda \mathrm{c} / \mathrm{V}$. The dissipation function will be essentially positive when there is enough structural damping. 
where $x, y, z$ are the rectangular cartesian coordinates of the element of mass $\delta \mathrm{m}_{2}$ anc the summation covers all the elements of mass for which the expression in the bracket does not vanish. The complete coefficient A contains, in addition, the corresponding virtual inertie or reverse ${ }^{\text {rs }}$ acceleration derivative.

When $q_{\text {, and }} q_{\mathrm{g}}$ are normal coordinates the coefficient $A_{r s}=A$ vanishes: ${ }^{-g}$ the same is true when $q$ is a normal coordinate and $q_{\mathrm{S}}$ is one of the general rigid body freedoms. This follows from the fact that motion in any normal mode can persist independently of any other motion.

Fon a cantilever wing with rigid support at the root we may usually neglect the chordwise and spanwise displacements in the flutter. Then the structural contribution to Ars is

$$
s_{\mathrm{rS}}=\sum_{\mathrm{m}} \frac{\partial \mathrm{z}}{\partial \mathrm{q}_{\mathrm{n}}} \frac{\partial \mathrm{z}}{\partial \mathrm{q}_{\mathrm{S}}} \delta_{\mathrm{m}_{0}}
$$

Further, when the wing is straight and not swept back or forward and it is assumed that each fore-and-aft strip moves as a rigid body this expression can be reduced to the form (see the list of symbol's)

$$
\frac{s^{2}-\frac{2 s}{4 x}}{c_{0}^{4} x}=\int \ln (n) d
$$

where

$$
\begin{aligned}
& a \operatorname{rs}(q)=m \frac{d y}{d q_{r}} \frac{d y}{d q_{s}}\left(\frac{c}{c_{0}}\right)^{2} \\
& \therefore p\left(\frac{d g}{d q_{s}} \frac{d v}{d q_{s}}+\frac{d s}{d q_{s}} \frac{d v}{d q_{s}}\right)\left(\frac{c}{c_{0}}\right)^{3} \\
& \therefore j \frac{a w^{2}}{d q_{2}} \frac{a}{d q_{s}}\left(\frac{c}{c_{0}}\right)^{4}
\end{aligned}
$$

\section{(b) Cross or Compound Demping Coefficients}

These are mainly or wholly of aerodynamic origin and the array of the coeficients is, in general, not symmetric, i.e., $\mathrm{B}_{\mathrm{rs}} ; \mathrm{B}_{\mathrm{Sr}}$ in general. We may, if we please, analyse these coupling coefficients into symmetric and skew-symmetric parts (see equations $5 A, 5 B$ ) and we may call these pure dampings and gyrostatic terms respectively, This analysis, however, does not appear to be particularly helpful.

\section{For}

3

If we assune that the virtual inertias are the same for a finite air speed and for still air then $q_{p}$ and $q_{\text {. }}$ should be normal coordinates for oscililations in still alr.

$x=$

To avoid circumlocution we shall henceforward call this a type C
vinge 
For the type $\mathrm{C}$ wing the general formula for an aerodynamic damping coefficient is

$$
\frac{B_{r s}}{e v c_{0}^{3} l}=\int Q_{r s}(\eta) d \eta
$$

where

$$
\begin{aligned}
& \beta_{r s}(\eta)=l_{\mathrm{w}} \frac{\mathrm{d} s}{\mathrm{dq}_{\mathrm{r}}} \frac{\mathrm{d} \mathrm{s}}{\mathrm{dq_{ \textrm {S } }}}\left(\frac{\mathrm{c}}{\mathrm{c}_{0}}\right) \\
& +\left(\ell_{\alpha} \frac{d j}{d q_{r}} \frac{d \eta}{d q_{s}}-m_{w} \frac{d j}{d q_{s}} \frac{d q}{d q_{r}}\right)\left(\frac{c}{c_{0}}\right)^{2} \\
& -\frac{m_{k}}{\alpha} \frac{d \gamma}{d q_{r}} \frac{d \vartheta}{d q_{s}}\left(\frac{c}{c_{0}}\right)^{3} \text {. }
\end{aligned}
$$

This expression is equivalent to that given in Appendix 3 of $\mathrm{R}$. and $\mathrm{M}$. $1904^{6}$.

\section{(c) Cross or Compound Stiffress Coefficients}

When normal coordinates are used these coefficients are entirely of aerodynamic origin and their array is, in general, not symmetric. For a type C wing

$$
\frac{c_{r s}}{\rho v^{2} c_{0}^{2} l}=\int C_{r s}(\eta) d \eta
$$

where, in accordance with R. and M. $1904^{6}$,

$$
\begin{aligned}
C_{r s} & =l_{\alpha} \frac{d j}{d q_{r}} \frac{d \vartheta}{d q_{s}}\left(\frac{c}{c_{0}}\right) \\
& -m_{\alpha} \frac{d \vartheta}{d q_{r}} \frac{d l}{d q_{s}}\left(\frac{c}{c_{0}}\right)^{2} .
\end{aligned}
$$




\section{Comparison of Inertial and Aerodynamic Couplings}

When normal coordinates are used the inertial couplings are zero and in certain instances a comparison of the expressions for the inertial and aerodynamic couplings shows that the latter also vanish. More generally, the comparison may enable us to show that certain aerodynamic couplings are small.

The special uniform wings mentioned in $\$_{2}$ provide examples of what has been said. Here $c / c_{0}$ is unity, the various coefficients are constants, and

$$
\frac{d \mathcal{L}}{d q_{r}}=k_{r} \frac{d \nu}{d q_{r}}
$$

where $k_{r}$ is constant for all $r$; this expresses the similarity of the normal añd torsional displacements in every natural mode. Hence

$$
\begin{aligned}
& \frac{s^{A s}}{c_{0}^{4 \ell}}=\left\{m_{r} k_{s}+p\left(k_{r}+k_{s}\right)+j\right\} \int \frac{d \ell}{d q_{r}} \frac{d \eta}{d q_{s}} d \eta \ldots(30)
\end{aligned}
$$

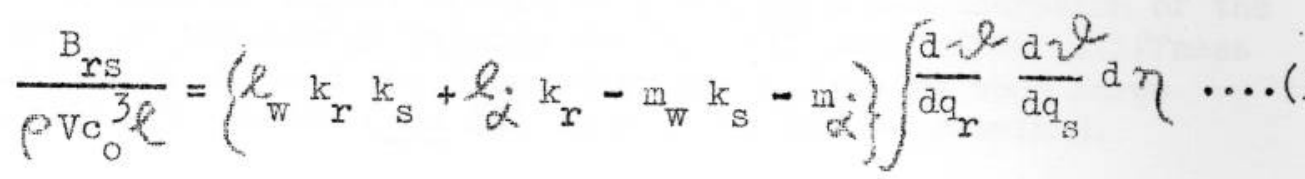

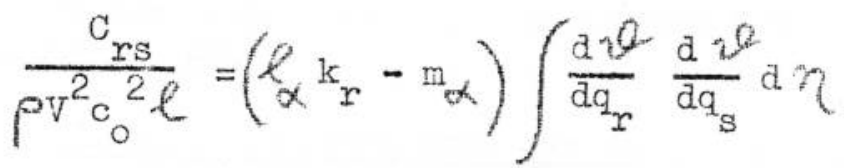

and all three coefficients vanish when

$$
\int \frac{d y}{d q_{r}} \frac{d \psi}{d q_{s}} d \eta=0 .
$$

\section{Principles Governing the Choice of Freedoms}

Suppose that a critical flutter speed $V$ has been calculated on the assumption that a particulor set of freedoms is effective and let it be enquired whether some other freedom, F say, should be included in the calculation. The complete answer to the question is given by repeating the whole calculation with the new freedom $F$ introduced and comparison of the new and original critical speeds and nodes of displacement. This method is usually extremely laborious and it is most desirable to find an easier basis of judgment. It is here suggested that judgment may be based on a consideration of the possible influence of $F$ upon the energy balance at the critical speed $V_{c^{*}}$. If it can be shown that the introduction of $F$ can neither ada nor subtract more than a relatively very small amount of energy then $F$ may safely be neglected. 
The irfluence of $F$ upon the energy balance will certainly be small when the amplitude of $F$ is very small. We may estimate the amplitude of $F$ by forning the Lagrangian equation of motion corresponding to $F$ and then assume that the other generalised coordinates have the values already calculated at the critical speed; this approximation will be justified if the amplitude of $F$ is in fact small. The amplitude of $F$ will then be given by

$$
\begin{aligned}
& \text { (Sum of coupling terms reversed) } \\
& \text { (Impedance in } F \text { at the flutter } \\
& \text { frequency) }
\end{aligned}
$$

Clearly the sum of the coupling terms will certainly be small when all the coupling coefficients are small and it may be possible to see that this is so wi.thout calculating these coefficients. The impedance will be large when the flutter frequency (in the absence of freedom F) differs widely from the natural frequency for $F$ alone, calculated at the wind
speed $V$ (since the direct stiffness coefficient for $F$ may depend on $V$ ). We conclude that it will not usually be safe to neglect $F$ when its proper frequency is near that of the flutter. On the other hand, when the proper frequency of $F$ is much higher than that of the flutter the impedance will be very large and the amplitude of $F$ will be very small;

When the amplitude of $F$ is neither large nor very smail the decision to retain or reject should be based on a consideration of the sensitiveness of the energy balance to F. If some of the stiffness couplings between $F$ and the other degrees of freedom show large skew-symmetric components (see S4) $_{4}$ then it should be retained.

\section{Illustrative Examples on the Choice of Freedoms}

A few simple examples are added here to illustrate the principles
an $5 \%$. put forward in 87 .

\section{In what circumstances can the aileron coordinate neglected in wing flutter?}

The stiffness coupling derivatives $I_{\psi}$ and $H_{\varphi}$ (zero) are widely different ${ }^{2}$; likewise $\mathrm{M} /$ and $\mathrm{H}_{\Theta}$ are widely different? Hence the equation of energy shows that movement of the aileron may feed in much energy to the system, as is otherwise obvious. Consequently the aileron coordinate $\xi$ must be retained unless it can be shown that the amplitude in 3 is very small. This will be true when either or both of the following conditions are satisfied:-

(i) The aileron impedance is very high. This can only be attained by providing the aileron with a very stiff elastic constraint (irreversible control).

(ii) All the coupling coefficients affecting the aileron hinge inoment are extremely small. These are the two aileron products of inertia, and the aerodynamic derivatives $\mathrm{H}_{\phi}, \mathrm{H}_{\dot{\theta}}, \mathrm{H}_{\theta}$. The latter will all be small for a certain location of the aileron hinge and the products of inertia can be made very small by suitable disposition of masses. 
(b) Must we bring in overtone modes in considering the flexuraltorsional flutter of a cantilever wing not carrying large concentrated massess?

We have seen in $\mathrm{S} 2$ that for a uniform cantilever wing with a certain special but quite reasonable elastic specification the tro gravest modes are strictly uncoupled to the higher modes. For other straight cantilever wings of normal proportions the couplings will be small, although not zero. A.lso the impedances in the overtone modes at the flutter frequency will be large on the great disparity in frequency. We conclude that the amplitudes in the overtone modes will be very small and that only minor errors ir the critical flutter speed will result from neglect of such modes.

\section{(c) $\frac{\text { Fiexural-torsional flutter of a cantilever wing carrying }}{\text { a. large concentrated mass }}$}

inertigl coupl If we take one of the two gravest modes the inertial coupling coefficient with any higher overtone mode is zero. In the expression for this 'product of inertia' the displacements at the large mass are clearly heavily 'weighted', but these displacements are not heavily weighted in the expressions for the aerodynamic couplings. Thus we have evidently no right to assume that the aerodynamic couplings of the modes are small just because the inertial couplings are zero. Hence the higher overtone modes can onily be neglected when their impedances at the flutter frequency are very large, i.e., when the disparity of frequency is large. Hence we must expect to find it necessary to retain some of the lower overtone modes beyond.
the first. 


\section{PART II}

\section{METHODS EOR PREDICTING FLUTTER}

\section{CHARACTERISTICS}

\section{Non-Dimensional Form of the Equations of Motion}

It is convenient to reduce the equations of motion to a standard non-dimensional form. When all the displacements are proportional to exp ( $\lambda t)$ the complete set of dynamical equations in the selected degrees of freedom can be written in the matrix notation

$$
\left(A \lambda^{2}+B V A+D V^{2}+E\right) q=0
$$

where $A$ is the inertia matrix

BV " " damping matrix

$E$ " " matrix of the elastic stiffnesses

$D^{2}$ " " matrix of the aerodynamic stiffnesses.

We shall now suppose that the $m$ dynamical coordinates $q$ are all non-dimensional (e.g., angles). Then, if $\rho$ and $\sigma$ are air and material densities and $e$ is a typical linear dimension, we may define a set of non-dimensional matrices of coefficients by the equations

$$
\begin{aligned}
& A=\ell^{5} \mathrm{a} \\
& B=\rho \ell^{4} \mathrm{~b} \\
& D=\rho e^{3} \mathrm{a} \\
& \mathrm{E}=\sigma \ell^{3} \mathrm{U}^{2} \mathrm{C}
\end{aligned}
$$

where $U$ is one of the velocities of propagation of elastic waves in a. typical material of construction. Next, introduce the auxiliary non-dimonsional variables

$$
\begin{aligned}
& \eta=\sqrt{\frac{\rho}{\sigma}} \\
& \xi=\left(\frac{u}{\eta v}\right)^{2} \\
& r=\frac{e \lambda}{\eta v} .
\end{aligned}
$$

The equation (34) can now be rewritten in the non-dimensional form

$$
\left(a^{2}+\eta^{b} x+a+\xi e\right) q=0
$$

A.t a critical flutter speed and for the flutter component we can put

$$
r= \pm i \omega
$$


and the equation to be satisfied is

$$
\left(\cdots a w^{2} \pm i \eta b w+d+\xi e\right) q=0 .
$$

The problem consists in finding $\xi$ and $\omega$ so that the set of linear scalar equations represented by the foregoing matrix equation shall be compatible. Methods for doing this are described below.

10. The Orthodox Method of Solution ${ }^{2}$.

We regard $\eta$ and the matrices $a, b, d$ and $e$ as known constants. If frequency-dependent derivatives are used this implies that some estimate of the flutter frequency parameter has already been made.

The conditionfor compatibility of the scalar equations represented by (42) is the 'determinantal equation'

$$
\Delta(x, s)=0
$$

where the expression on the left is a polynomial in $h$, in general of degree 2in, whose coefficients are functions of $\xi$. At a critical value of this equation is satisfied by the pair of equal and opposite roots $t i e w$. Accordingly the parts of $\Delta$ containing even and odd powers of $\omega$ vanish separately and we derive a peir of equations which we may write

$$
\begin{aligned}
& \Delta_{1}(\omega, 2, \xi)=0 \\
& \Delta_{2}\left(\omega, \frac{\omega}{\xi}\right)=0 .
\end{aligned}
$$

The result of eliminating to from these is

$$
T_{n-1}(5)=0
$$

where ${ }^{\mathrm{n}-1}$ is the penultimate Routhian test function and $n$ is the degree in $\supset$ of the deteminantal equation. The last is a polynomial equation for $\xi$ and when its roots have been found the critical flutter speeds can be obtained from (40). Finally, the flutter frequency and. the complex modal ratios can be calculated.

It is worthy of remark that the test function always vanishes when the relative density parameter $\eta$ is zero. Thus $T_{n-1}$ contains a power of $\eta$ as a fector and when this is removed we are left with a polynomial in ' ? whose 'constant' term is the asymptotic form of the test function for low relative air density. Since the critical values of $\xi$ are then independent of $\eta$, equation $(40)$ shows that the 'equivalent: or indicated critical flutter speeds are independent of the densities. It appears that modern heavily loaded aircraft are in or near this asymptotic condition.

The orthodox method is systematic and yields all the desired information. Its arawback is that it becomes unworkably laborious when the number of freedoms is greater than 3 . 
11. The Method of R.A. Frazer ${ }^{8}$

Frazer writes the equations $(46)$ and $(47)$ as $^{\text {Ix }}$

where

$$
\begin{aligned}
& f(x, y)=0 \\
& g(x, y)=0
\end{aligned}
$$

$$
\begin{aligned}
& x=-\omega^{2} \\
& y=\xi .
\end{aligned}
$$

and

Since $x$ and $y$ are both real they could be found from the real intersections of the curves (49) and (50). Frazer shows that the full expressions for the functions $f$ and $g$ can easily be calculated from expressions in partial fraction form containing the values of $\triangle(K, \xi)$ corresponding to a special set of points $(x, y)$. These are the intersections of a standard set of straight lines satisfying the conditions

$$
\begin{aligned}
& \text { (a) No two are parallel } \\
& \text { (b) No three are concurrent. }
\end{aligned}
$$

Much labour can be saved by use of this method when the number of freedoms is large. It is to be regarded as a special development of the classical method, designed to minimise labour.

Frazer and Bratt have proposed mechanical and electrical methods for the description of the curves (49) and (50).

\section{Methods Based on the Use of Matrices}

The determination of the roots $K$ for a given 5 can be made to depend on the problem of finding the latent roots of a certain matrix (Duncan and Collar9,10). An iterative method for finding the latent roots without expanding the characteristic determinant is given by Duncan and Collar and other treatments are described by Hermann and Dörr11. It is not considered that these methods have yet been developed to the stage where they would be advantageous from the aspect of labour saving in flutter problems.

\section{The Method of Duncan, Collar and Lyon 7}

This method is based on the fact that the mechanical impedance of the system is zero at the critical flutter speed and for simple harmonic applied forces having the frequency of the flutter. The method consists, in effect, in calculating the impedance for a number of assumed air speeds and frequencies and determination of the condition of zero impedance by interpolation. Since the impedance is found merely by solving a set of simultaneous linear equations with known coefficients, the calculations are relatively simple. The method has the further advantage that the modes of oscillation in the flutter are obtained incidentally from the calculations.

\section{It $\ldots \ldots \ldots \ldots$}

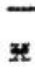

Frazer does not use the special reduction to the non-dimensional form adopted here, but his method is essentially as stated. 
It is conventent to male the calculations in the following manner. Arbitraily assign the amplitude of one dynamical coordinate (say, put $q_{6}=1$ ) and use the dynemical equations corresponding to free motion in the other coordinates to calculate the ermlitudes of these for the assumed air meed and frequency; this requires only the solution of a set of simuteneous linear equations whose coefficients are, in general, co plex on account of the presence of the damping terms. The cmplitudes of 2.1 the dynamical coordinates are now known axd when these are inserted in the equation for the coordinate q. the expression on the Ieft hand side is ccual to the amplitude of the generalised force $\theta_{n}$ whicis must be applied in order to maintain the assumed notion- This is the inpedance, in general complex, since $q_{n}$ is unity the vanishing 0 th complox impedance implies two conditions and it thus becones possible to find both the critical flutter speed and frequency by interpolation when the inpedance has been colculated for a few pairs of values.

The cuick success of this method evidently depends on making a good preliminaty guess os estimate of the critical flutter speed and frequency.

\section{Another Iriverse Method.}

We may proceed exactly as in the last method until we reach the Pinel dyamica'- equation corresponding to the assigned coordinate $a^{\circ}$. Honever, the expression on the left hand side of the equation is equated to zero (free motion) while $\xi$ in this is regarded es uninomo We shall thus derive a value $g^{\prime}$ for $\xi$, in general compiex; the condition to be satisfied is

$$
5-5=0
$$

which is equivalent to a pein of real equations. The critical values of $\frac{}{2}$ and $\omega$ are to be obtained $3 y$ trial snd interpolation, as in the proiousty described method. 


\section{IIST OF PRINCIPAL SYMBOLS USED}

The symbols are in alphabetical order and the Greek letters follow the Roman.

An accent indicates the transposed of a matrix.

A dot above a symbol indicates its time rate of change. The numbered references are to equations.

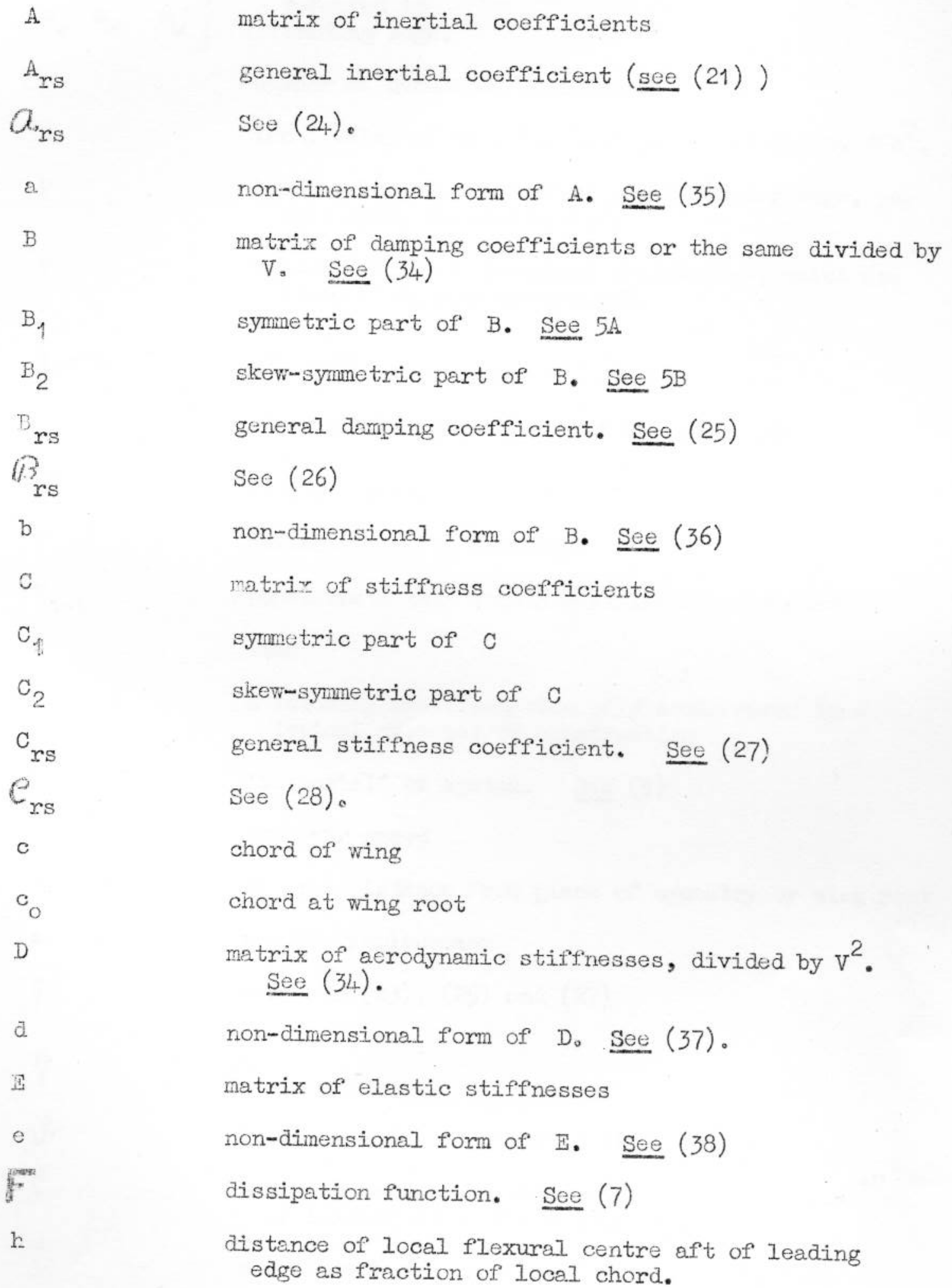


local value of moment of inertia about leading edge, per unit span, divided by $\sigma c^{4}$

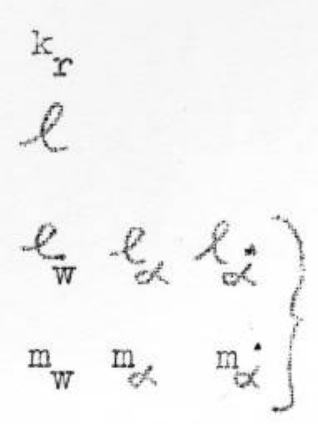

m

m

$\mathrm{p}$

q

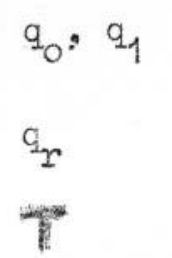

T

$T_{n-1}$

t

U

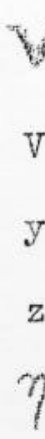

$\eta$

$\zeta$

$\xi$
See (29)

distance from wing root to reference section or other typical linear dimension. See also $n$.

Local non-dimensional aerodynamic derivative coefficients referred to leading edge.

number of dynamical coordinates

local value of mass per unit span, divided by $\sigma_{c}^{2}$.

local value of mass moment about leading edge, per unit span, divided by of $c^{3}$.

column matrix of dynamical coordinates, which are taken to be non-dimensional.

See (16)

$r^{\text {th }}$ dynamical coordinate or its amplitude

kinetic energy of system

periodic time of flutter

penultimate test function in Routh's sequence

time

a velocity of propagation of elastic waves in a typical material of construction

'potential' of system. See (9)

true air speed

lateral distance from plane of symmetry or wing root normal displacement

$y / e$ in (23), (25) and (27)

$\sqrt{\frac{2}{\sigma}}$

local twisting or pitching displacement

downward normal displacement of leading edge, divided by $\mathrm{c}_{0}$.

non-dimensional speed coefficient. See (40) 
$-20-$

$x$

a. non-dimensional frequency parameter. See (41)

$\lambda$

$\mu$

0

б

ses coefficient of $t$ in $\exp (\lambda t)$

real part of $\lambda$

air density

density of typical material of wing

coefficient of imaginary unit in $\lambda$ or $\mathrm{K}$, See (16), (43); 


\section{IIST OF REFERENCES}

No.

1

3

4

5

6

7
Author

Gates, S.B.

Frazer, R.A. and.

Duncan, W.J.

Frazer, R.A. and

Duncan, W.J.

Frazer, R.A. and

Duncen, W.J.

Duncan, พ.J.

Duncan, W.J.

$$
\begin{aligned}
& \text { Duncan, W.J., } \\
& \text { Coltar, A.R., } \\
& \text { and }
\end{aligned}
$$

Lyon, H.M.

Frazer, R.A.

Duncan, W.J. and.

Collar, A.R.

$$
\begin{gathered}
\text { Frazer, R.A., } \\
\text { Duncan, W.J. } \\
\text { and }
\end{gathered}
$$

Collai, A.R.

Herman, A.

and.

Dörr, J.
Title etc.

The Torsion-Flexure Oscillations of Tro Connected Beams. Phil.Mag., Jan. 1928.

The Flutter of Aeroplane Wings. (A.R.C. Monograph).

R. \& M. 1155, Aug. 1928.

Conditions for the Prevention of Flexural-Torsional Flutter of an Elastic Wing.

R. \& M. 1217, Dec。 1928 ,

The Flutter of Monoplanes, Biplanes and Tail Units. (A.R.C. Monograph) R. \& M. 1255, Jan. 1931.

Note on the Flutter of Complicated Systems. A.R.C.7630 (Unpublished) April, 1944.

The Representation of Aircraft Wings, Tails and Fuselages by Serni-Rigid Structures in Dynamic and Static Problems. R. \& M. 1904, Feb. 1943.

Oscillations of Elastic Blades and Wings in an Airstream. R. \& M. 1716, Jan. 1936 。

Bi-variate Partial Fractions and their Applications to Flutter and Stability Problems. Proc, Roy. Soc., A, Vol. 185, p. $465,1946$.

Matrices Applied to the Motions of Damped Systems. Phil. Mag, , Ser. \%, vol. 19, p.197: Feb, 1935.

Elementary Matrices. Cambridge University Press, 1938.

Forschungsbericht

No. 1769, Feb. 1943.

Translation by Miss S.W. Skar

'Methods of Investigating Flutter' Behaviour when there are a Number of Degrees of Freedom! A.R.C. 10,439, Narch, 1947. 